\title{
Critical exponents of directed percolation measured in spatiotemporal intermittency
}

\author{
Peter Rupp, Reinhard Richter, and Ingo Rehberg \\ Physikalisches Institut, Experimentalphysik V, Universität Bayreuth, D-95440 Bayreuth, Germany \\ (Received 11 January 2002; revised manuscript received 31 October 2002; published 25 March 2003)
}

\begin{abstract}
An experimental system showing a transition to spatiotemporal intermittency is presented. It consists of a ring of hundred oscillating ferrofluidic spikes. Four of five of the measured critical exponents of the system agree with those obtained from a theoretical model of directed percolation.
\end{abstract}

DOI: 10.1103/PhysRevE.67.036209

PACS number(s): 05.45. $-\mathrm{a}, 47.54 .+\mathrm{r}, 47.52 .+\mathrm{j}$

\section{INTRODUCTION}

Intermittency was observed in hydrodynamics as a precursor to turbulence (see, e.g., Ref. [1]). For dissipative dynamical systems without spatial degrees of freedom intermittency was first modeled by ordinary differential equations and iterative maps [2]. Spatiotemporal intermittency (STI) is a further development of this concept for spatially extended systems and was introduced by Kaneko [3] in the mid 1980's [4]. It is characterized by patches of ordered and disordered states fluctuating stochastically in space and time [5]. This behavior has been observed in many systems. Theoretical approaches have been made in a large variety of systems ranging from partial differential equations, such as the damped Kuramoto-Sivashinsky equation [6,7] or the complex Ginzburg-Landau equation [8-10], over stochastical partial differential equations [11] to coupled map lattices (CML) $[3,12,13]$ and probabilistic cellular automata [14].

In 1986 Pomeau [15] suggested that the onset of chaos via STI might be analogous to directed percolation (DP) [16]. Such processes are modeled as a probabilistic cellular automaton with two states per site, associated with the laminar and chaotic patches in the case of STI. One of the main features of DP is the presence of an absorbing state, which corresponds to the laminar state. The absorbing state prevents the nucleation of chaotic domains within laminar domains. DP model predicts some universal properties of STI. In particular, the fraction of chaotic domains is expected to grow with a power law $\epsilon^{\beta}$, where $\epsilon$ measures the distance from threshold. The correlation length decreases with $\epsilon^{-\nu_{s}}$, as does the correlation time with $\epsilon^{-\nu_{t}}$, and the critical distribution of the laminar lengths is determined by $l^{-\mu_{s}}$ and by $t^{-\mu_{t}}$ for the laminar times.

Some experimental tests of the conjecture [15] in quasi-onedimensional systems have been made [8,17-24]. A short summary of these and the relevant exponents $[25,26]$ for the comparison with the DP model are given in Table I. However, the statement that "... there is still no experiment where the critical behavior of DP was seen" by Grassberger [27] still seems to be true. Thus in this paper an experimental approach to this old problem is presented.

The system introduced consists of a ring of ferrofluidic spikes excited by an external magnetic field. Ferrofluids, also called magnetic fluids, are a colloidal suspension of ferromagnetic nanoparticels. The fluid is superparamagnetic [28]. The idea for using this fluid was motivated by the fact that a single peak of ferrofluid can show chaotic oscillations under external driving of a magnetic field $[29,30]$. We introduce here a system where about 100 of these oscillating peaks are coupled by magnetic and hydrodynamic interactions. They exhibit changes in peak height of about $10 \%$ and variations in wavelength $\lambda$ of about $50 \%$. This system is advantageous because of its short response times and the easy control of the excitation.

The paper is organized as follows. In Sec. II we describe the experimental setup, the procedure of the measurement, and the data extraction methods. In Sec. III the quantitative results are presented, i.e., the critical exponents $\beta, \nu_{s}, \nu_{t}$, $\mu_{s}$, and $\mu_{t}$. Finally in Sec. IV the results are discussed and an outlook to further investigations is given.

TABLE I. Experiments and results in quasi-one-dimensional systems.

\begin{tabular}{|c|c|c|c|c|c|c|c|c|c|}
\hline Authors & Year & Experiment & Size & $T_{0}(\mathrm{~s})$ & Geometry & $\beta$ & $\nu_{s}$ & $\nu_{t}$ & $\mu_{s}$ \\
\hline Ciliberto et al. [17] & 1988 & RB convection & 20 & 10 & Annular & & 0.5 & & $1.9 \pm 0.1$ \\
\hline Daviaud et al. $[8,18]$ & 1990 & $\mathrm{RB}$ convection & 40 & 2 & Linear & $0.3 \pm 0.05$ & $0.5 \pm 0.05$ & $0.5 \pm 0.05$ & $1.6 \pm 0.2$ \\
\hline Daviaud et al. $[8,18]$ & 1990 & RB convection & 30 & 2 & Annular & & 0.5 & 0.5 & $1.7 \pm 0.1$ \\
\hline Michalland et al. [19] & 1993 & Viscous fingering & 40 & 1.5 & Linear & $0.45 \pm 0.05$ & 0.5 & & $0.63 \pm 0.02$ \\
\hline Willaime et al. [20] & 1993 & Line of vortices & 15 & 5 & Linear & & & 0.5 & \\
\hline Degen et al. [21] & 1996 & Taylor-Dean & $20(90)$ & 1.5 & Linear & $1.30 \pm 0.26$ & $\approx 0.64$ & $\approx 0.73$ & $1.67 \pm 0.14$ \\
\hline Colovas et al. [22] & 1997 & Taylor-Couette & $30(70)$ & 0.5 & Linear & & & & \\
\hline Bottin et al. [23] & 1997 & Plane Couette & & & Linear & & & & \\
\hline Vallette et al. [24] & 1997 & Fluid fronts & 40 & 0.5 & Linear & & & & \\
\hline Jensen (theory) $[25,26]$ & 1999 & Directed percolation & & & & $0.276486(8)$ & $1.096854(4)$ & $1.733847(6)$ & 1.748 \\
\hline Present paper & & Ferrofluidic spikes & 108 & 0.08 & Annular & $0.3 \pm 0.05$ & $1.2 \pm 0.1$ & $0.7 \pm 0.05$ & $1.7 \pm 0.05$ \\
\hline
\end{tabular}




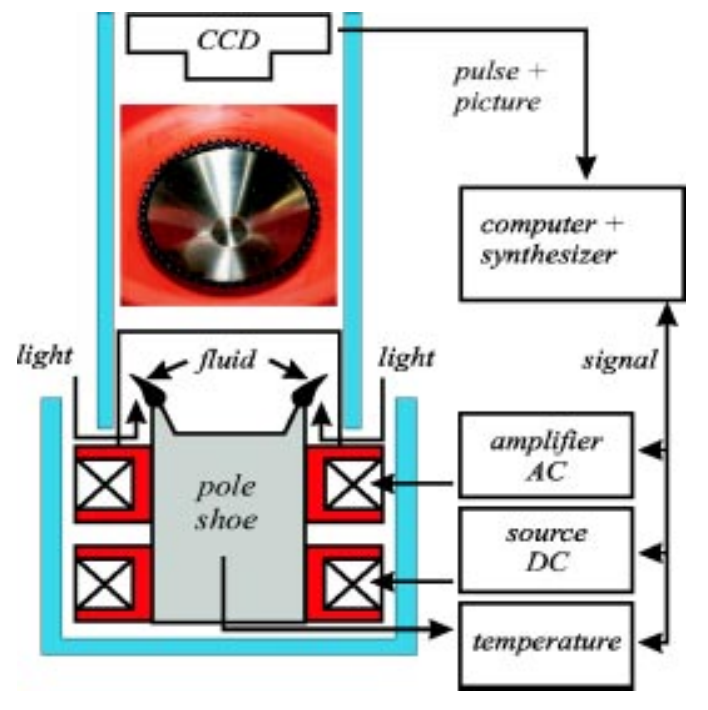

FIG. 1. Sketch of the experimental setup.

\section{EXPERIMENT}

The experiment is based on the Rosensweig instability [28]. This instability is observed in a horizontal layer of magnetic fluid, when a threshold of the vertically oriented magnetic field is surpassed. The flat surface becomes unstable and a pattern of liquid spikes emerges. In case of an inhomogeneous field the wavelength of this pattern can be controlled by the magnetic field $H$. The wavelength scales with the gradient of the magnetic field divided by a basic field at the undisturbed surface of the fluid. A larger gradient emphasizes small peaks and suppresses large ones. Thus, in order to generate as many spikes as possible, the setup of the experiment consists of a cylindrical electromagnet with a sharp edge (Fig. 1). The magnetic fluid is trapped by the inhomogeneous magnetic field at this edge of the magnetically soft iron core. In that way the 40-mm diameter of the pole shoe supports a ring of up to 130 spikes of magnetic fluid as indicated by the picture in Fig. 1.

The magnetic fluid used in this experiment is EMG901 from Ferrofluidics ${ }^{\mathrm{TM}}$, a fluid based on magnetite $\mathrm{Fe}_{3} \mathrm{O}_{4}$ with isoparafin as carrier fluid. At $20^{\circ} \mathrm{C}$ EMG901 has a density $\rho=1.53 \mathrm{~g} \mathrm{~cm}^{-3}$, a surface tension $\sigma=29 \mathrm{mN} \mathrm{m}^{-1}$, a dynamical viscosity $\eta=25 \mathrm{mPa}$ s and a susceptibility $\chi=3$.

The ring of spikes is recorded with a charge-coupled device (CCD)-camera mounted above the pole shoe. The electromagnet consists of a bias coil and an excitation coil. The bias coil is provided with a direct current of $I=1.0$ A to keep the magnetic fluid in its place. The excitation coil is driven by an alternating current, phase locked with the camera frequency, providing a stroboscopic jitter free recording on long time scales. The alternating current can be adjusted between 0 and 4.1 A. In this interval the number of spikes ranges from 60 to 130. For the amplitudes used in the experiment, 108 spikes have been observed.

To keep the viscosity and the surface tension constant, the fluid is temperature controlled to $12.5 \pm 0.03{ }^{\circ} \mathrm{C}$ by cooling the pole shoe. To prevent the evaporation of the isoparafin, the volume around the edge of the pole shoe is sealed with a glass plate to provide long term stability.

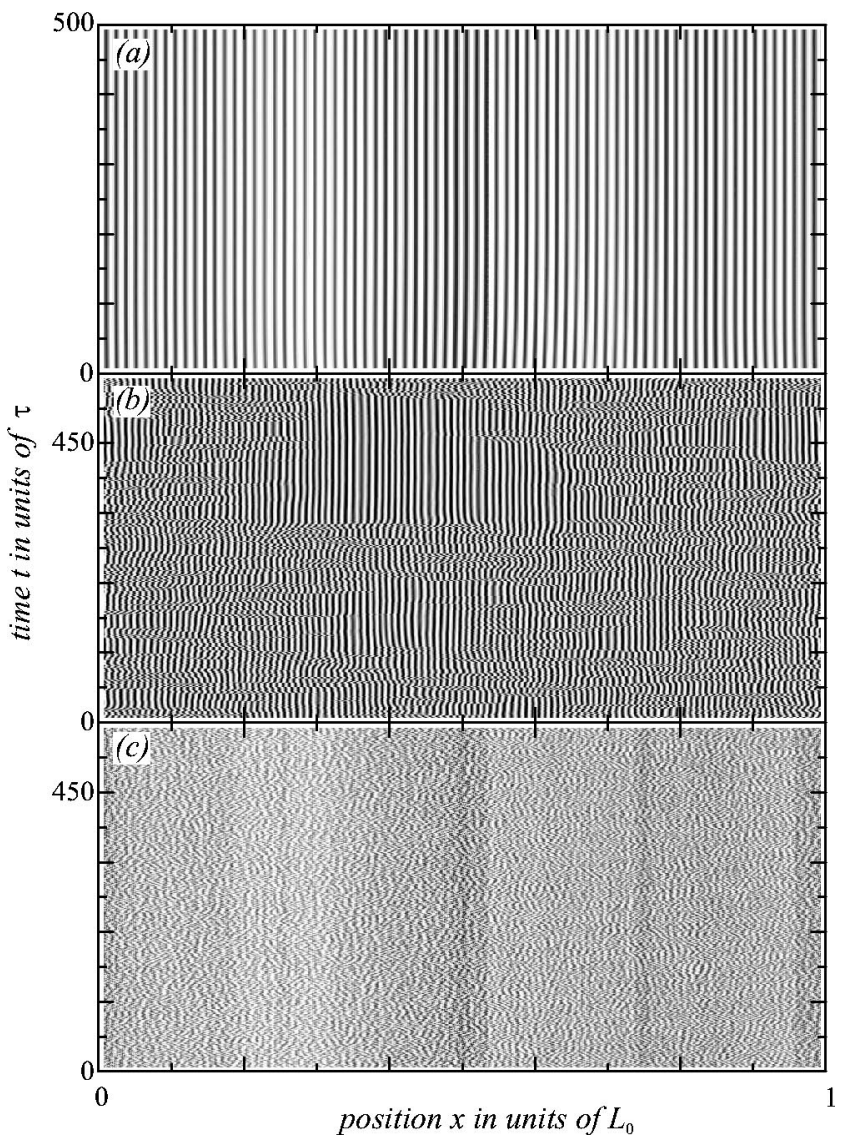

FIG. 2. Space-time plots of the different states of the system. (a) $I_{\mathrm{ex}}=2.8 \mathrm{~A}$, laminar state; (b) $I_{\mathrm{ex}}=3.0 \mathrm{~A}$, spatiotemporal intermittency; (c) $I_{\mathrm{ex}}=3.6 \mathrm{~A}$, chaotic state. 500 excitation periods are shown. The position $x$ is normalized over the size of the ring.

The spatiotemporal behavior is investigated by observing the ring with the CCD camera. To extract the wavelength and amplitude of the spikes as a function of space, we define a ring of interest around the center of the pole shoe, which is covering the ring of spikes. It is divided into 1024 segments. The average of the gray values within each segment represents the amplitude. That way we get a spatial resolution of around ten segments per peak. This reduction of the twodimensional image to a single line scan can be done in real time with a frequency of $12.5 \mathrm{~Hz}$.

In Fig. 2(a) 500 of such scans of a laminar state are shown in space and time, where dark regions correspond to high amplitudes. The driving frequency of the excitation field is $f_{\text {ex }}=12.5 \mathrm{~Hz}$, as mentioned above. The period $\tau=1 / f_{\text {ex }}$ is used to scale the time. Due to this stroboscopic recording the oscillations of the spikes cannot be seen.

For the measurements a current of $1 \mathrm{~A}$ is applied to the bias coil and a constant volume of the magnetic fluid is dropped on the edge of the pole shoe. After a waiting time of $2 \mathrm{~h}$ thermal equilibrium is reached. Then the second coil is provided with a sinusoidal excitation signal of an amplitude of $I_{\mathrm{ex}}=4.05 \mathrm{~A}$, driving the system into the fully chaotic regime similar to the one indicated in Fig. 2(c). That state is 

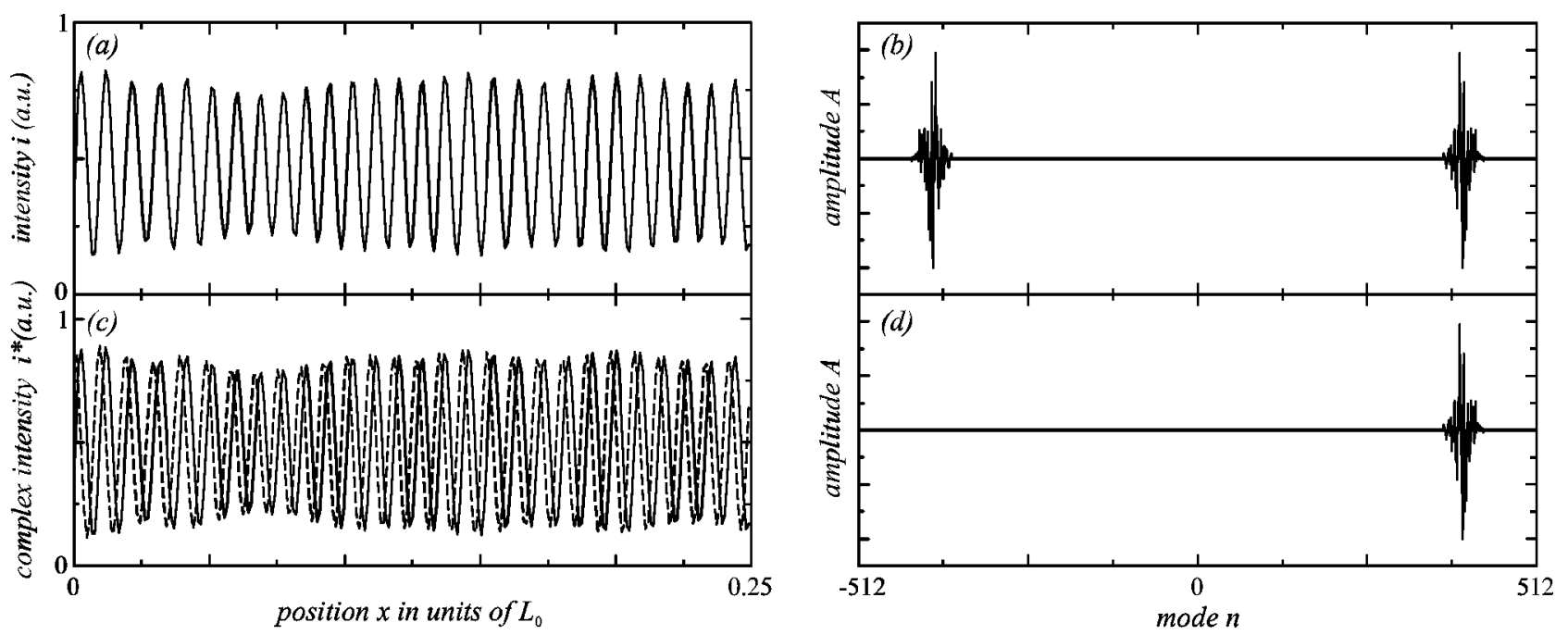

FIG. 3. Complexification of the data (see text). The left hand side displays the data in real space [(a),(c)], the right hand side the corresponding graphs in Fourier space $[(b),(d)]$.

the basis for a quench to a lower excitation value corresponding to the STI regime [Fig. 2(b)], which is then analyzed subsequently. The recording of the data starts after a waiting time of $\approx 1800 \tau$, when the transients following the quench have died out. The data are recorded for 2000 excitation periods $\tau$. For higher excitation amplitudes the laminar state [Fig. 2(a)] becomes intermittent in space and time [Fig. 2(b)] and eventually chaotic [Fig. 2(c)].

For a quantitative analysis of the transition a reliable detection of the regular domains is fundamental. Different criteria for the distinction of the regular and chaotic domains have been proposed $[5,17-24]$. Because of the strong variations of the wavelength of our system we have selected a wavelength criterion, which is based on the local wavelength $\lambda(x, t)$. To obtain $\lambda(x, t)$ we use a method called complexification, which is based on the Hilbert transformation

$$
i_{x}^{*}=\mathcal{F}^{-1}\left\{H(n) \mathcal{F}\left\{i_{x}\right\}\right\},
$$

where $i_{x}$ is the real intensity at position $x$ [Fig. 3(a)], $i_{x}^{*}$ is the complex intensity at $x$ [Fig. 3(c)], and $H(n)$ is the Heaviside function in Fourier space [Figs. 3(b) and 3(d)]. By this method a zero imaginary part is added to every real value [see Fig. 3(a)]. Then the data are transformed to Fourier space, where the amplitudes corresponding to negative wave numbers are eliminated [Figs. 3(b) and 3(d)]. With a backward transformation to real space, every value has a nonzero imaginary part [Fig. 3(c), dashed line]. $\lambda$ is subsequently calculated as the phase difference between neighboring values in real space [Fig. 4(a)]. In the last step the relative changes of the local wavelength

$$
\Delta=\frac{\left|\lambda_{t+1}-\lambda_{t}\right|}{\lambda_{t}}
$$

are calculated. To get a clear distinction between regular and irregular domains, changes in $\Delta$ which are larger than 0.01 are counted as irregular, whereas smaller changes belong to regular domains [Fig. 4(b)]. The threshold value $\Delta_{c}$ is de- rived from the distribution of $\Delta$ for the fully laminar case which is presented in Fig. 5 by a solid line. There are no larger variations than $1 \%$. Smaller values of $\Delta$ are artefacts of the recording technique and thus are suppressed. For comparison, the dashed (dotted) lines give the distribution of $\Delta$ for the intermittent (chaotic) states. The calculations of the exponents are robust to changes of the threshold of up to $50 \%$. This variation of the binarization threshold corresponds to changes in the resulting critical exponents within the statistical errors. In Fig. 6 we demonstrate the application of the above described procedure to the intermittent data. Figure 6(a) gives the raw data, (b) displays the local wavelength, and (c) the relative change $\Delta$ after binarization. The quantitative analysis of STI described below is based on this binarized information. To get a better signal-to-noise ratio we average the results over six independent runs of the experiment, which include six refills of the apparatus with fresh fluid.

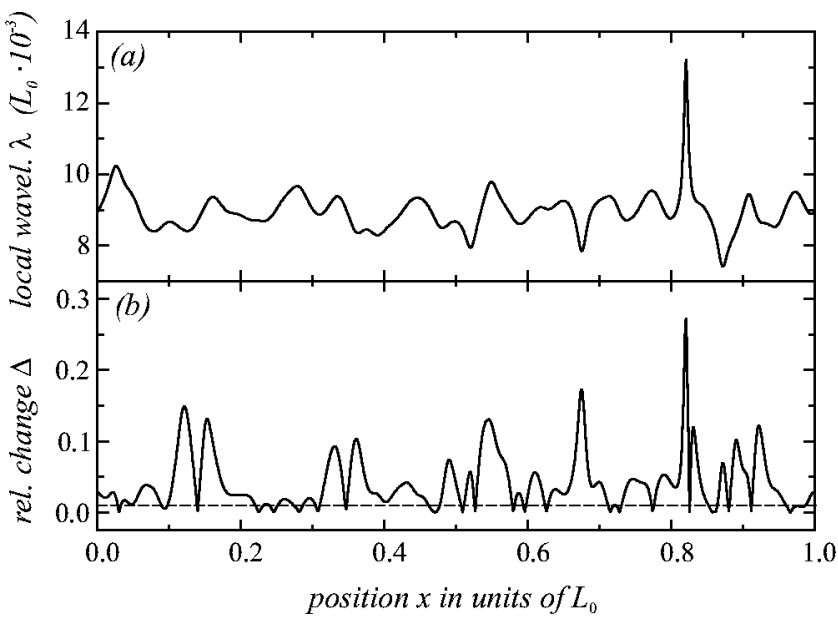

FIG. 4. (a) Local wavelength $\lambda$ calculated from Fig. 3(c) in units of $L_{0}$. (b) Relative change of local wavelength $\Delta$. The dashed line corresponds to the threshold of 0.01 taken from Fig. 5. 


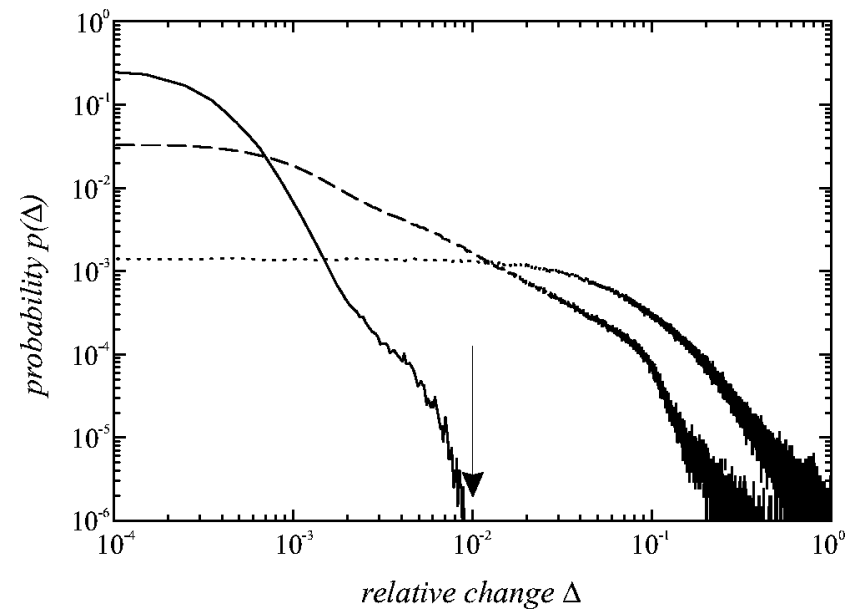

FIG. 5. Distributions of the relative change of wavelength $\Delta$ for three different $I_{\mathrm{ex}}$ : solid line $I_{\mathrm{ex}}=2.8 \mathrm{~A}$ corresponding to Fig. 2(a), dashed line $I_{\mathrm{ex}}=3.0$ A corresponding to Fig. 2(b), and dotted line $I_{\mathrm{ex}}=3.5$ A corresponding to Fig. 2(c). The cutoff of the distribution for the fully laminar state gives the threshold of the binarization $\Delta_{c}=0.01$, as depicted by the arrow.

\section{EXPERIMENTAL RESULTS}

At low excitation amplitudes $I_{\mathrm{ex}} \ll 3.0 \mathrm{~A}$ the system is completely regular [Fig. 2(a)] showing 108 spikes. Slight spatial variations of the wavelength $\Delta<0.01$ remain constant in time. In Fig. 2(b) at $I_{\mathrm{ex}}=3.0 \mathrm{~A}$ irregular fluctuations are apparent, which we consider as a manifestation of STI. A fairly clear distinction between regular and irregular domains can be made in this image even by naked eye. Further increase of $I_{\text {ex }}$ leads to a spreading of the irregular domains engulfing the regular regions, until finally the whole system is chaotic [Fig. 2(c)].

As an order parameter for STI we take the time-averaged chaotic fraction $\gamma$, which is the ratio of chaotic regions to the length of the system. This ratio is averaged over the 2000 excitation periods $\tau$. Its variation with the control parameter $I_{\mathrm{ex}}$ is shown in Fig. 7. The error bars represent the variance of the chaotic fraction.

Close to the onset of STI the mean chaotic fraction is expected to grow with a power law

$$
\gamma \sim\left(I_{\mathrm{ex}}-I_{c}\right)^{\beta}
$$

The solid line in Fig. 7 is a fit to our data, using $I_{c}, \beta$, and an offset representing background noise as adjustable parameters. The threshold value determined in this way is $I_{c}=3.0$ $\pm 0.05 \mathrm{~A}$ and the exponent $\beta=0.3 \pm 0.05$ is in agreement with the theoretical expectation for DP $\beta=0.276486(8)$ [25].

Another way to characterize the regular domains is to look at the mean laminar expansion in space $\ell$ and time $T$. First we define the laminar lengths $l$ as the number of consecutive regular segments between two chaotic ones divided by the total number of segments at a certain time $t_{0}$. The

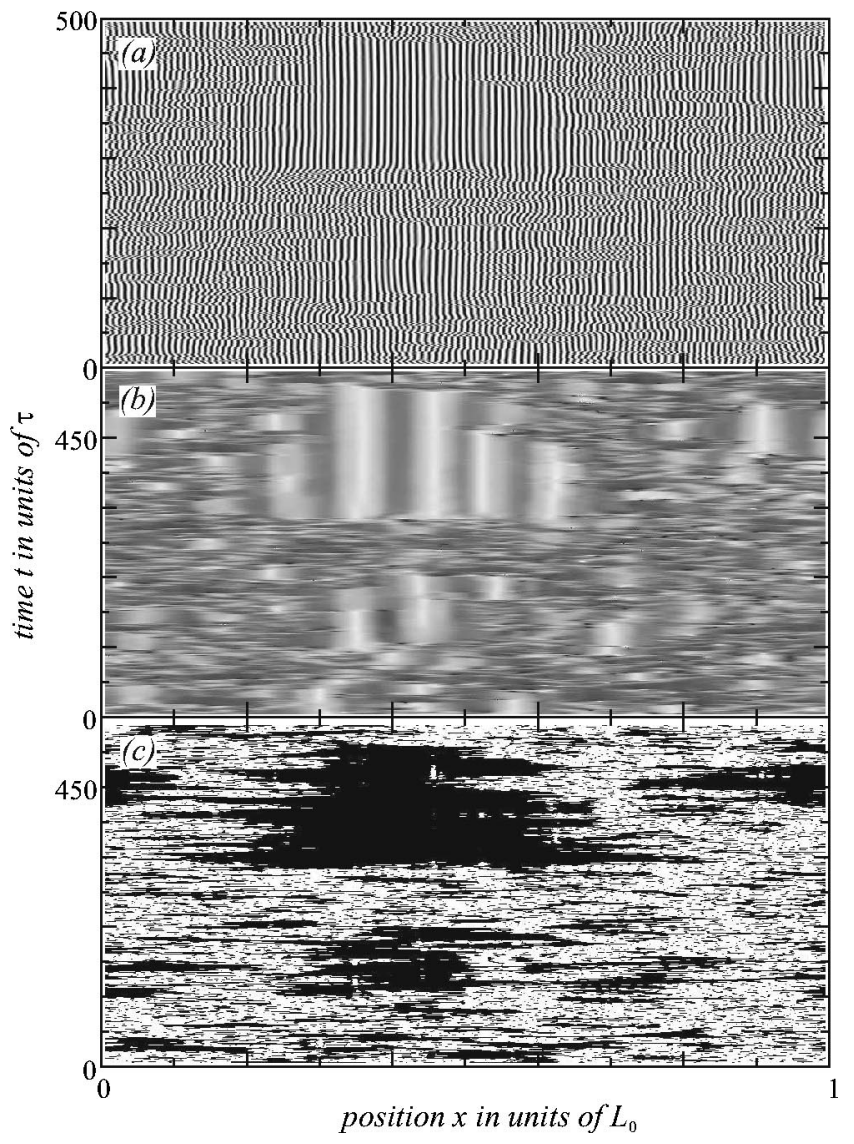

FIG. 6. $x$-t $t$-plots at different states of the data processing. (a) Raw data; (b) same section as in (a) after transforming the data. White corresponds to large local wavelengths, black to small ones. (c) Final step: the relative change of local wavelength over two subsequent periods $\Delta$ is calculated and binarized. The black areas are defined as regular $(\Delta<0.01)$, the white are chaotic $(\Delta$ $>0.01)$.

laminar times $t$ are the number of segments between two chaotic ones at a certain position $x_{0}$. The averages of these numbers are displayed in Fig. 8 as the function of the normalized control parameter

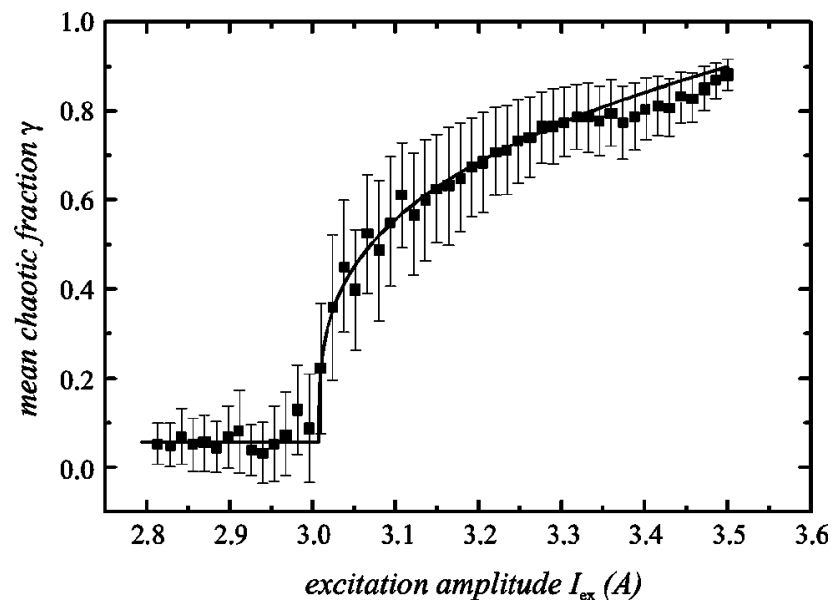

FIG. 7. The mean chaotic fraction $\gamma$ versus excitation amplitude $I_{\text {ex }}$. The solid line is a power law fit. The error bars represent the statistical errors. 


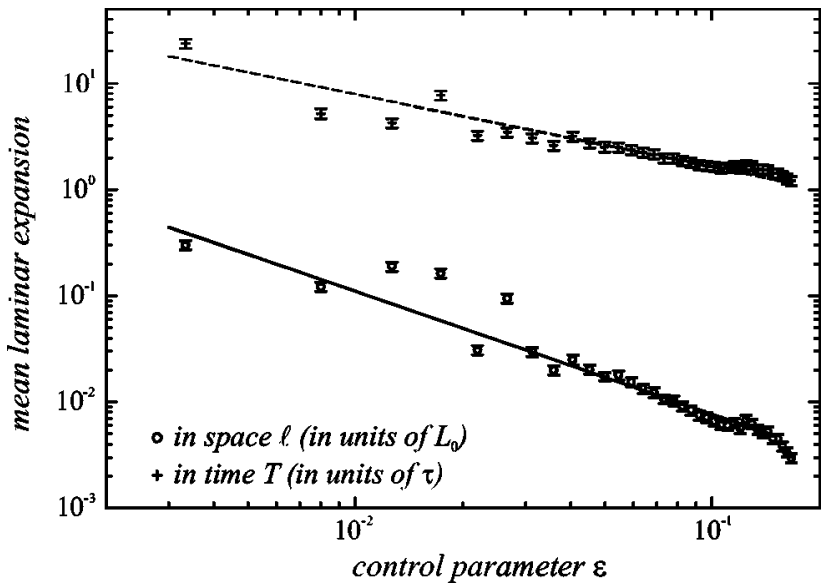

FIG. 8. The mean laminar expansion in space $\ell$ and in time $T$ versus control parameter $\epsilon$. The lines are power law fits. The error bars represent statistical errors.

$$
\epsilon=\frac{I_{\mathrm{ex}}}{I_{c}}-1 .
$$

Both parameters decay with a power law:

$$
\begin{aligned}
& \ell \sim \epsilon^{-\nu_{s}^{*}}, \\
& T \sim \epsilon^{-\nu_{t}^{*}}
\end{aligned}
$$

and the fit yields the exponent $\nu_{\mathrm{S}}^{*}=1.2 \pm 0.2$, which is represented by the solid line and $\nu_{t}^{*}=0.7 \pm 0.1$, which is represented by the dashed line. Only data in the range of 0.03 $<\epsilon<0.1$ are taken into account. For smaller $\epsilon$ a finite size effect is obvious: Following Cross and Hohenberg [31] the characteristic length of the regular domains $\ell$ must be much smaller than the system size 1 . For $\epsilon>0.1$ the system is no longer intermittent, but rather chaotic.

In Fig. 9 the distributions of the laminar domain length and time for $\epsilon=0$ are presented. At the threshold the distribution should follow a power law for both the distribution of

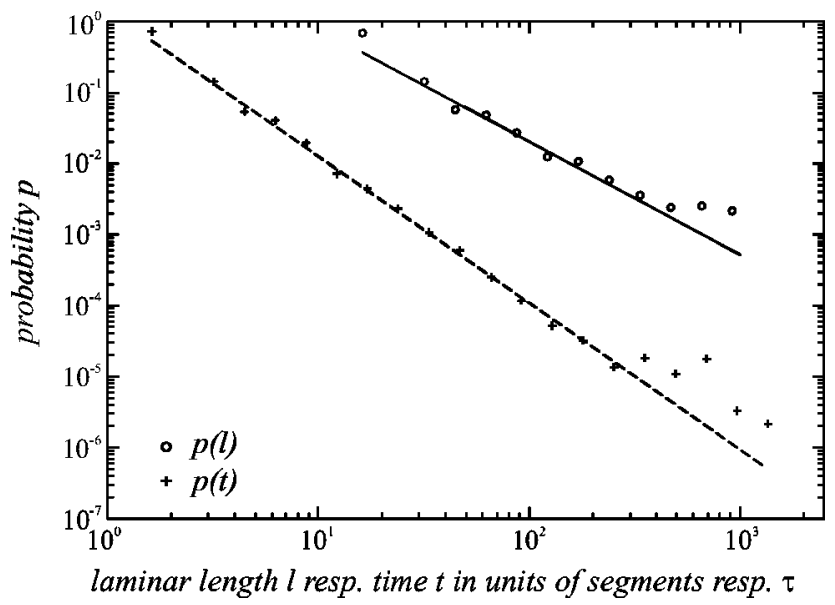

FIG. 9. Distribution of the laminar domain length $l$ and time $t$ for $\epsilon=0$. The solid lines are power law fits. To suppress the statistical fluctuations the distributions are logarithmically binned.
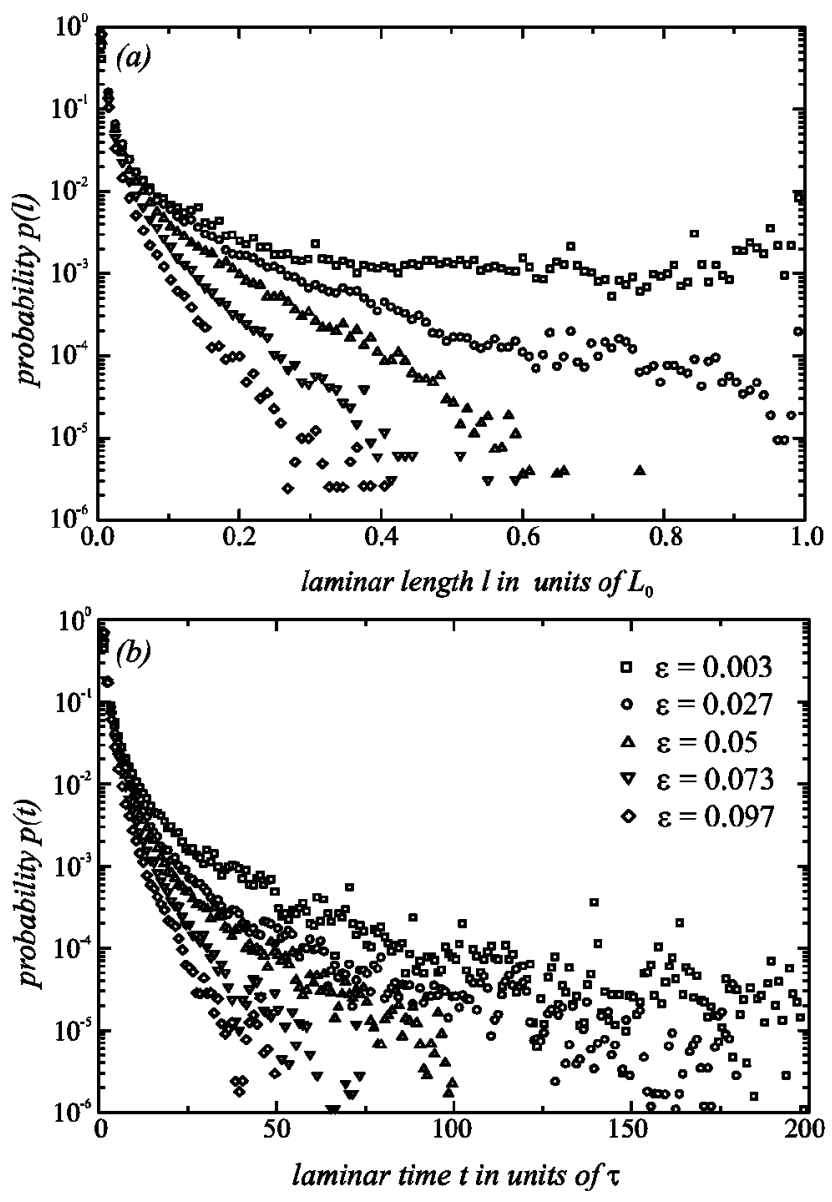

FIG. 10. Distributions of the laminar length $l$ (a) and the laminar time $t$ (b) for $\epsilon>0$. The legend in (b) holds for both figures.

the laminar domain lengths $l$ and times $t$. To suppress the statistical fluctuations the values are logarithmically binned. The solid line represent the power law fit for the distribution of the lengths (7) and for the times (8):

$$
\begin{aligned}
& p(l) \sim l^{-\mu_{s},} \\
& p(t) \sim l^{-\mu_{t}} .
\end{aligned}
$$

We obtain $\mu_{s}=1.7 \pm 0.05$, in agreement with the theoretical value $\mu_{s}=1.734$ and $\mu_{t}=2.1 \pm 0.1$, in accordance with the theoretical value $\mu_{t}>2.0$.

For $\epsilon>0$ the power law has a cutoff at the correlation length (time) of the system and an exponential tail with a decay length equal to the correlation length $\xi_{\text {decay }}$ (time $\left.\theta_{\text {decay }}\right)$ (Fig. 10).

Both parameters should grow with the same power law as the mean expansions $\ell$ and $T$ in Eqs. (5) and (6). This behavior can be seen in Fig. 11. The lines correspond to power law fits with the exponents $\nu_{s}=1.1 \pm 0.2$ and $\nu_{t}=0.62$ \pm 0.14 . The large errors are due to the statistical fluctuations of the distributions and difficulties in the definition of the cutoff length.

The distributions cannot be described by a simple power law. A more complicated distribution function has been suggested, 


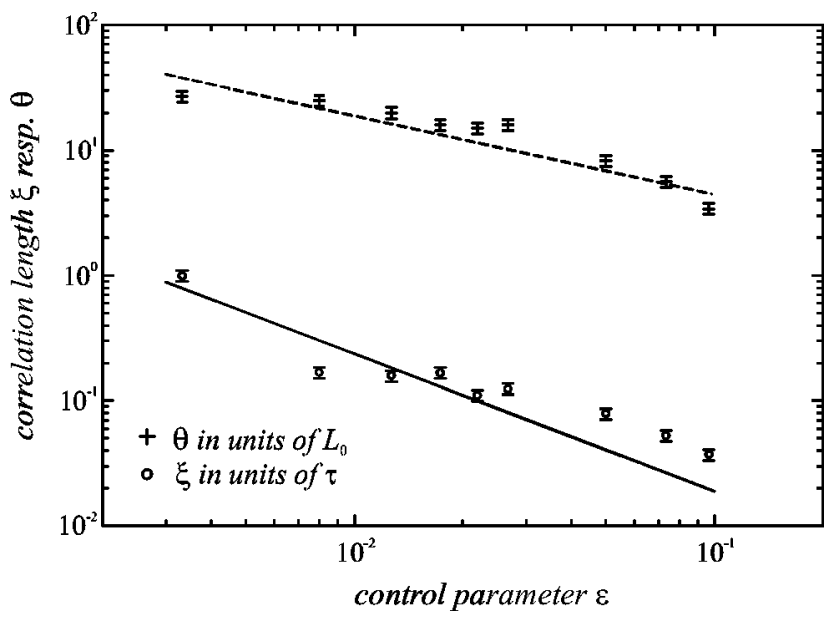

FIG. 11. The correlation length $\xi$ and time $\theta$ derived from the exponential decay of the distributions (Fig. 10) versus control parameter $\epsilon$. The lines are power law fits. The error bars represent statistical errors.

$$
p(l)=\left(A l^{-\mu}+B\right) e^{-l / l_{\text {decay }}}
$$

in Ref. [17]. The solid line in Fig. 12 is a fit to this empirical distribution function for $\epsilon=0.019$ with $l_{\text {decay }}=0.17$. It shows clearly that the power law is now replaced by a function more reminiscent of an exponential decay.

\section{DISCUSSION}

To conclude, we have presented an experimental system exhibiting STI. In contrast to all previous experiments, which are autonomous ones, our system is periodically driven. We have measured the critical exponents $\beta, \nu_{s}, \nu_{t}$, $\mu_{s}$, and $\mu_{t}$ for the mean chaotic fraction $\gamma$, the mean laminar length $\ell$, the mean laminar time $T$, the correlation length $\xi_{\text {decay }}$ and time $\theta_{\text {decay }}$, and the laminar length and time distribution functions for $\epsilon \approx 0$. Four of the five exponents agree with the theoretical expectation derived from a DP model within our experimental resolution. Considering the simplicity of the underlying discrete model, the fact that the theory is applicable only near $I_{c}$, the complexity of our experiment, and the fact that our apparatus has a finite size, this concordance seems truly remarkable.

The fact that the parameter $\nu_{t}$ is far from the expected value needs further discussion. One difference between the underlying model and our experiment might be the nature of the absorbing state. In fact, the absence of a truly absorbing state seems to be common to all experimental results so far

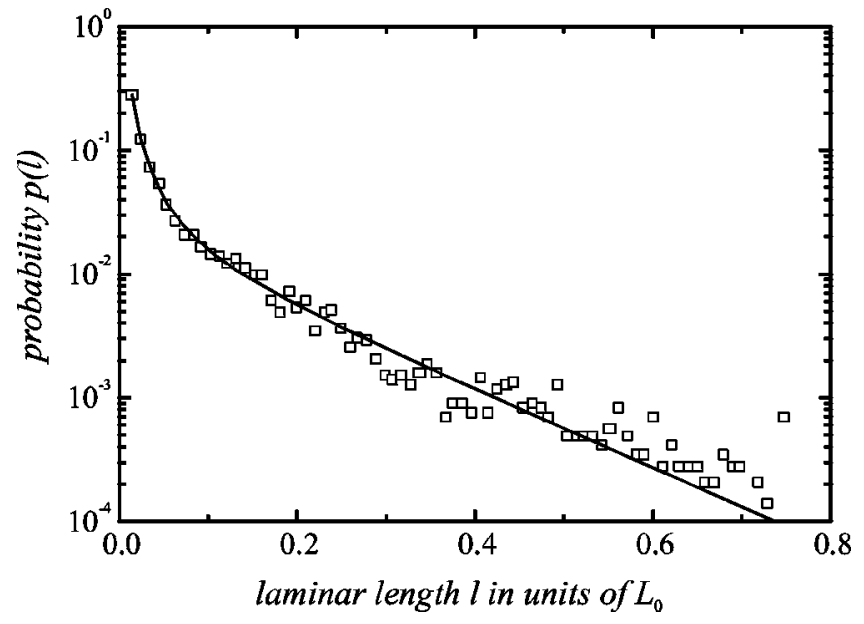

FIG. 12. Distribution of the laminar domain length $l$ for $\epsilon$ $=0.019$. The solid line stems from Eq. (9).

[32]. Following the ideas presented in that book the allowance for a continuum of states between the absorbing and the active one might result in a more realistic description of the experimental situation.

Another way to soften the assumption of a truly absorbing state is the introduction of stochastic mechanisms permitting the nucleation of chaotic domains. In DP implementing a weak external field that creates chaotic domains in a pure laminar neighborhood with a certain probability $[25,33]$ does this. As long as the probability of the creation processes is small the critical behavior of DP is only slightly disturbed. If the probability becomes too large the universality is destroyed. The stochastic field could be an anologon to the "background noise" of our experimental apparatus.

Alternatively, traveling solitonlike structures have added to CML and DP [34]. The interaction of these structures might lead to chaotic domains nucleating in laminar regions, which again softens the assumption of a truly absorbing state and leads to the breakdown of universality in DP.

If any of these ideas will be able to explain the behavior of the measured decay times is subject to further investigation.

\section{ACKNOWLEDGMENTS}

The authors would like to thank Hugues Chaté and Haye Hinrichsen for helpful discussions. One of us (R.R.) would like to thank Victor Steinberg for inspiring contributions. Deutsche Forschungsgemeinschaft through Re588/12 has financially supported the experiments.
[1] D.J. Tritton, Physical Fluid Dynamics, 2nd ed. (Clarendon Press, Oxford, 1988).

[2] Y. Pomeau and P. Manneville, Commun. Math. Phys. 74, 189 (1980).

[3] K. Kaneko, Prog. Theor. Phys. 74, 1033 (1985).

[4] For a more general review see Refs. [35] and [32].

[5] H. Chaté and P. Manneville, in Turbulence: A Tentative Dic- tionary, edited by P. Tabelling and O. Cardoso (Plenum Press, New York, 1995), p. 111.

[6] H. Chaté and P. Manneville, Phys. Rev. Lett. 58, 112 (1987).

[7] U. Frisch, Z.S. She, and O. Thual, J. Fluid Mech. 168, 221 (1986).

[8] F. Daviaud, J. Lega, P. Berge, P. Coullet, and M. Dubois, Physica D 55, 287 (1992). 
[9] R.J. Deissler, J. Stat. Phys. 40, 371 (1985).

[10] M. van Hecke, Phys. Rev. Lett. 80, 1896 (1998).

[11] M.G. Zimmermann, R. Toral, O. Piro, and M. SanMiguel, Phys. Rev. Lett. 85, 3612 (2000).

[12] H. Chaté and P. Manneville, Physica D 32, 409 (1988).

[13] P. Grassberger and T. Schreiber, Physica D 50, 177 (1991).

[14] H. Chaté and P. Manneville, Physica D 37, 33 (1989).

[15] Y. Pomeau, Physica D 23, 3 (1986).

[16] A good introduction to DP is found in Ref. [36], for a more recent review of most aspects of DP see Ref. [25].

[17] M. Caponeri and S. Ciliberto, Physica D 58, 365 (1992).

[18] F. Daviaud, M. Bonetti, and M. Dubois, Phys. Rev. A 42, 3388 (1990).

[19] S. Michalland, M. Rabaud, and Y. Couder, Europhys. Lett. 22, 17 (1993)

[20] H. Willaime, O. Cardoso, and P. Tabeling, Phys. Rev. E 48, 288 (1993).

[21] M.M. Degen, I. Mutabazi, and C.D. Andereck, Phys. Rev. E 53, 3495 (1996).

[22] P.W. Colovas and C.D. Andereck, Phys. Rev. E 55, 2736 (1997)

[23] S. Bottin, O. Dauchot, and F. Daviaud, Phys. Rev. Lett. 79, 4377 (1978).

[24] D.P. Vallette, G. Jacobs, and J.P. Gollub, Phys. Rev. E 55, 4274
(1997).

[25] H. Hinrichsen, Adv. Phys. 49, 815 (2000).

[26] I. Jensen, J. Phys. A 32, 5233 (1999).

[27] P. Grassberger, in Nonlinearities in Complex Systems, Proceedings of the 1995 Shimla Conference on Complex Systems, edited by S. Puri (Narosa, New Delhi, 1997).

[28] R.E. Rosensweig, Ferrohydrodynamics (Cambridge University Press, Cambridge, 1985).

[29] T. Mahr and I. Rehberg, Physica D 111, 335 (1998).

[30] R. Friedrichs and A. Engel, Eur. Phys. J. B 18, 329 (2000).

[31] M.C. Cross and P.C. Hohenberg, Rev. Mod. Phys. 65, 851 (1993).

[32] K. Kaneko and I. Tsuda, Complex Systems: Chaos and Beyond (Springer, Berlin, 2000).

[33] H.K. Janssen, Ü. Kutbay, and K. Oerding, J. Phys. A 32, 1809 (1999).

[34] T. Bohr, M. van Hecke, R. Mikkelsen, and M. Ipsen, Phys. Rev. Lett. 86, 5482 (2001).

[35] P. Manneville, Dissipative Structures and Weak Turbulence (Academic Press, Boston, 1990).

[36] W. Kinzel, in Percolation Structures and Processes, edited by G. Deutscher, R. Zallen, and J. Adler (Adam Hilger, Bristol, 1983), Vol. 5, pp. 425-445. 